\title{
Early Re-brightenings in GRB Afterglows As Signatures of Low-to-High Density Boundary
}

\author{
P. H. Tam ${ }^{a, 1}$, C. S. J. Pun ${ }^{a}$, Y. F. Huang ${ }^{b}$, K. S. Cheng ${ }^{a}$ \\ ${ }^{a}$ Department of Physics, The University of Hong Kong, Pokfulam Road, Hong Kong, China. \\ ${ }^{b}$ Department of Astronomy, Nanjing University, Nanjing 210093, China.
}

\begin{abstract}
The association of long gamma-ray bursts (GRBs) with star forming regions and the idea of massive stars as progenitors of GRBs are widely accepted. Because of their short lifetimes, it is very likely that massive stars are still embedded in dense molecular clouds when they give birth to GRBs. Stellar winds from GRB progenitors can create low-density bubbles with sizes and densities strongly depending on the initial ambient density. A boundary between the bubble and the dense molecular cloud must exist with the density at the boundary increasing from that of the bubble to that of the outer cloud. We have calculated the lightcurves of the afterglows in such environments with three regions: the stellar wind region, the boundary, and the molecular cloud. We show that the interaction between the cylindrical jet and the density boundary can result in a re-brightening of the afterglow occurring as early as $\sim 1$ day after the GRB. We compare our models with the optical afterglows of GRB 970508, GRB 000301C, and GRB 030226. We find that the values of our model parameters, including the radius of the wind bubble, the densities in the bubble and in the outer molecular cloud are within typical ranges.
\end{abstract}

\footnotetext{
${ }^{1}$ Corresponding author - e-mail: acthomas@graduate.hku.hk

This work has been accepted to be published in New Astronomy
} 


\section{Introduction}

The association of supernovae (SNe) with gamma-ray bursts (GRBs), of which a convincing example is GRB 030329 - SN 2003dh, gives strong supports for massive stars as progenitors of GRBs (Hjorth et al., 2003). It has been suggested that GRBs are located in star-forming regions (Holland \& Hjorth, 1999; Holland et al., 2001; Lamb \& Reichart, 2000) and most of the star formation in the universe occurs in molecular clouds. Several observational evidences of the presence of molecular clouds around GRB progenitors have been found. Galama \& Wijers (2001) analyzed a sample of eight GRB afterglows. They found X-ray evidence for column densities $N_{\mathrm{H}}=10^{22}-10^{23} \mathrm{~cm}^{-2}$ of gas around these GRBs. From dust destruction and survival of neutral Mg, they further constrained the clouds to be of masses $\gtrsim 10^{5} M_{\odot}$ and sizes within 10 to 30 parsecs. These values are consistent with measurements of Galactic giant molecular clouds (GMCs; Solomon et al. 1987). Moreover, Reichart \& Yost (2001) showed that dark bursts likely occur in clouds of similar sizes. Reichart \& Price (2002) further suggested that the bursts with detected optical afterglows also occur in GMCs and have shown that the results are consistent with observations statistically.

Observations reveal that GMCs $\left(M \sim 10^{5}-10^{6} M_{\odot}, R \sim 6-60 \mathrm{pc}, n_{\mathrm{H}} \sim 10^{2}-\right.$ $10^{3} \mathrm{~cm}^{-3}$ ) are highly inhomogeneous and contain a large number of clumps or dense cores $\left(M \lesssim 10^{3} M_{\odot}, R \sim 0.1-1 \mathrm{pc}, n_{\mathrm{H}} \sim 10^{4}-10^{5} \mathrm{~cm}^{-3}\right)$. These dense clumps or cores are believed to be the birth places of massive stars. In fact, such high density environments have been used to explain the afterglows of GRB 980519 and GRB 990123 (Dai \& Lu, 2000; Wang et al., 2000).

Jets in GRBs have been proposed to avoid large implied isotropic energy in some GRBs and to explain the rapid decay in some afterglow lightcurves (Rhoads, 1997, 1999;

Sari et al., 1999; Huang et al., 2000b, 2000c; Frail et al., 2001). However, almost all these discussions have assumed a conical geometry (i.e. keeping the half-opening angles of the jets largely constant, or allowing some lateral expansion), which we think may not be sufficient. Observations of some relativistic jets in radio galaxies, active galaxies, and "microquasars" show that they are very likely to be cylindrical (i.e. at large scales the 
cross-sectional areas of the jets are constant or at most expand laterally with sound speed; Perley et al., 1984; Biretta et al., 1999). Therefore it is necessary to explore the behaviors of cylindrical jets in addition to conical jets. Cheng et al. (2001) noted its significance and gave analytic as well as numerical calculations of the cylindrical jet model. Huang et al. (2002) showed that the afterglows of GRBs 970228, 970508, 971214, 980329, and 980703 could be fit by cylindrical jets propagating into uniform medium. Cylindrical jets propagating in free wind environments (i.e. $n \propto r^{-2}$ ) have also been analyzed (Ma et al., 2003).

The physics of stellar winds around the GRB progenitors has widely been considered in the literature (Chevalier \& Li, 1999, 2000; Ramirez-Ruiz et al., 2001; Wu et al., 2003). The strong wind blown out from a very massive star will dramatically affect the density distribution in the circumstellar medium. In a dense cloud $\left(n_{0} \sim 10^{5} \mathrm{~cm}^{-3}\right)$, the wind is able to drive a low density bubble, which evolves to a radius of about $1 \mathrm{pc}$ in $\approx 10^{5}$ years (Shull, 1980), beyond which situates a denser shell mainly consisting of swept-up materials. Dai \& Lu (2002) analyzed the hydrodynamics and emission features of GRB ejecta on a preburst environment with a sudden density jump.

The increasing number of GRB afterglow lightcurves observed enables us to model the fine details of their evolutions. One important behavior is the re-brightenings (or "bumps") in the optical afterglow lightcurves. They show excess flux compared to extrapolation from earlier data, deviating from the predictions of simple models which assume isotropic fireballs or collimated jets and homogeneous interstellar medium (ISM) or simple wind environment (i.e. $n \propto r^{-2}$ ). Many late-time bumps (e.g. GRBs 970228, 980326, and 011121) have been attributed to SN components because of the reddening of the observed spectra (Bloom, 2003; Bloom et al., 1999, 2002; Galama et al., 2000; Garnavich et al., 2003b). These late-time bumps are also argued to be due to dust echoes (Esin \& Blandford, 2000). On the other hand, GRB 970508 and GRB 000301C show early-time optical bump(s) with no significant color change in the spectra. A number of proposals to explain the early-bumps have been raised. For example, energy injections and gravitational microlensing have been suggested to explain the bump(s) in afterglows of GRB 970508 (e.g. Panaitescu et al., 1998; Chang et al., 2002) and GRB 000301C (Garnavich et al., 
2000) respectively. In this paper, we present a unified model to explain these two GRBs: a cylindrical jet, after pushing through the low density bubble, can produce the observed lightcurve bump when it encounters the molecular cloud. GRB 030226 is another burst which reveals a re-brightening during $t \sim 0.2-0.5$ day (Dai \& $\mathrm{Wu}, 2003$ ), thus can also be interpreted by our model.

The structure of this paper is as follows. We outline the models in section 2, emphasizing the three regions existed at the time of GRBs, i.e. the wind bubble region, the intervening region, and the molecular cloud. In section 3 we present numerical results showing the effects of the density boundaries on the optical lightcurves, which are characterized by observed bumps. We proceed in section 4 to compare our model with the optical afterglow lightcurves of GRBs 970508, 000301C, and 030226. Finally, we discuss our results and present our conclusion in section 5 .

\section{The Model}

\subsection{Density profile}

The strong wind blown out from a very massive star (OB-type main sequence) will drastically affect the density distribution in the circumstellar medium because of the large mass-loss rate and the fast wind (Castor et al., 1975; Weaver et al., 1977). They demonstrated that the stellar wind will create a low-density bubble. When the mass of swept-up interstellar gas is comparable to the wind mass, materials will accumulate at a certain radius, at where situates a high density shell. The shell will then expand gradually during the remaining life-time of the star. They showed that after $t \approx 10^{6}$ years, the bubble size can be about $20-30$ parsecs. The bubble is much smaller (about $0.1-1.0 \mathrm{pc}$ ) when the star is embedded in a dense cloud $\left(n_{0} \sim 10^{5} \mathrm{~cm}^{-3}\right.$; Shull, 1980). In this case, unshocked molecular gas remains outside the shell with a particle density typical of that within a dense cloud ( $n \sim 10^{3}-10^{5} \mathrm{~cm}^{-3}$ ). Ramirez-Ruiz et al. (2001) presented a detailed analysis of stellar winds from single Wolf-Royet stars. Ramirez-Ruiz et al. (2001) also considered the afterglows of GRBs resulting from the interactions between Wolf-Royet 
winds and jets of GRBs. They assumed that the jets of GRB have conical geometry and found that re-brightenings can occur in afterglows about 10 - 100 days after the GRBs. They suggested that this effect could explain the observed bumps in afterglows of GRB 970228, GRB 980326, and GRB 000911. However, the model cannot explain re-brightenings occurring in less than 10 days. For example, GRB970508 has a bump in the optical afterglow at $\sim 1$ day after the main burst.

We consider a simple model in this paper just to illustrate the effects of density boundaries on the afterglow lightcurves. In this model, the density $n_{1}$ is assumed to be constant for $R<R_{\text {rise }}$, where $R_{\text {rise }}$ represents the onset of the boundary between the stellar wind and the molecular cloud. We call this Region (1). This region corresponds to the region of nearly constant density in the simulated density profiles in Weaver et al. (1977) and Ramirez-Ruiz et al. (2001). We assume $R_{\text {rise }}$ to be larger than the deceleration radius $R_{0}$, where the afterglow phase starts. The density increases rapidly for a distance $d$ to a much higher value $n_{2}$. We designate the density rising zone Region (2) and the dense outer zone Region (3). Figure 1 illustrates the different regions in our density model of the GRB surroundings.

\subsection{Kinetic Equations of Cylindrical Jet}

The idea of cylindrical jets has been supported by many observations in systems other than GRBs. For example, jets in many radio galaxies are cylindrical and maintain constant cross sections on large scales. In addition, jets in many Herbig-Haro (HH) objects are cylindrical (e.g. Ray et al., 1996). Observations show that HH jets are poorly focused at first, but are collimated into cylinders at sufficiently late times.

Theoretically, black hole-accretion disk systems can naturally produce cylindrical jets (Shu et al., 1995; Krasnopolsky et al., 2003; Vlahakis \& Königl, 2003a, 2003b; Fendt \& Ouyed, 2004), with magnetic forces playing important roles in the collimation process. The poloidal component of a dipolar magnetic field varies as $B_{P} \propto r^{-3}$, where $r$ is the distance from the center. Fendt \& Ouyed (2004) showed that the motion of matter along the poloidal magnetic field lines will unavoidably produce a strong toroidal field 
component, which decays as $B_{T} \propto r^{-1}$. As a result, a magnetohydrodynamic (MHD) jet is dominated by the toroidal component $B_{T}$ at large length scales. This field then exerts an inward force on the MHD jet through "hoop stress" and provides the collimation. Many numerical results have shown that MHD jets are initially conical. After the acceleration process, their half opening angles become smaller and finally the jets become cylindrical ones (e.g. Krasnopolsky et al., 2003). In the case of GRBs, believed to occur in star formation regions, large density gradients in the circumburst regions may also play a role in collimation of the jets.

We therefore believe that the cylindrical jet model is worth exploring in the case of GRBs, especially when one of the most popular models now, the collapsar model (Woosley, 1993; Paczyński, 1998), suggests the black hole-accretion disk system as the progenitors of GRBs. Before calculating the afterglow emissions, we need a unified dynamical model to describe the evolution of GRB jets. In the internal-external shock model (Piran, 1999; Cheng \& Lu, 2001), afterglow is generated from the external shock at which the ejecta of GRB is slowed down due to their interactions with the surrounding materials. Such deceleration can be described by the following equation, which are valid for both the relativistic and non-relativistic phases (Huang et al., 1999a, 1999b):

$$
\frac{d \gamma}{d m}=-\frac{\gamma^{2}-1}{M_{\mathrm{ej}}+\varepsilon m+2(1-\varepsilon) \gamma m},
$$

where $\gamma$ is the Lorentz factor of the macroscopic motion of the shocked material, $m$ the rest mass of the swept-up ISM, $M_{\mathrm{ej}}$ the ejected mass from the progenitor, and $\varepsilon$ the radiation efficiency. Here we assume an adiabatic jet and adopt $\varepsilon=0$ throughout this paper (this is a good approximation as long as $\xi \ll 1$ ). The evolution of the distance from the progenitor $(R)$, the swept-up mass $(m)$ and the lateral radius $(a)$ of the cylindrical jet can be described by the following equations (Cheng et al., 2001):

$$
\begin{gathered}
\frac{d m}{d R}=\pi a^{2} n m_{p}, \\
\frac{d R}{d t}=\frac{\beta c}{1-\beta \cos \Theta}, \\
\frac{d a}{d t}=\frac{v_{\perp}}{\gamma(1-\beta \cos \Theta)}
\end{gathered}
$$


where $n$ is the number density of hydrogen atoms with mass $m_{p}$ in the circumburst medium, $t$ the observer's time, $\beta=\sqrt{1-1 / \gamma^{2}}, \Theta$ is the angle between jet axis and our line of sight, and $v_{\perp}$ the lateral expansion speed of the jet in the comoving frame respectively. Since observations have shown that the jets in other astrophysical objects are well-collimated (Perley et al., 1984; Biretta et al., 1999; also see Cheng et al., 2001), in this paper we will adopt $v_{\perp}=0$ (i.e. the cylindrical jets strictly maintain a constant cross-sectional area).

\subsection{Synchrotron Radiation}

The dominant radiation mechanism of the afterglows is synchrotron radiation from the accelerated shocked material. The observed flux density at frequency $\nu$ is given by (Huang et al., 2000a, 2000c)

$$
S_{\nu}=\frac{1}{\gamma^{3}(1-\beta \cos \Theta)^{3}} \frac{1}{4 \pi D_{L}^{2}} P^{\prime}[\gamma(1-\beta \cos \Theta) \nu],
$$

where $D_{L}$ is the luminosity distance, and $P^{\prime}\left(\nu^{\prime}\right)$ is the synchrotron power at $\nu^{\prime}$ in the local frame given by Rybicki \& Lightman (1979):

$$
P^{\prime}\left(\nu^{\prime}\right)=\frac{\sqrt{3} e^{3} B^{\prime}}{m_{e} c^{2}} \int_{\gamma_{e, \min }}^{\gamma_{e, \max }}\left(\frac{d N_{e}^{\prime}}{d \gamma_{e}}\right) F\left(\nu^{\prime} / \nu_{c r}^{\prime}\right) d \gamma_{e},
$$

in which $B^{\prime}$ is the local magnetic field strength, $e$ the electron charge, $\gamma_{e, \max }=10^{8} / \sqrt{B^{\prime}(\mathrm{G})}$, $\gamma_{e, \text { min }}=\xi_{e}(\gamma-1)\left(m_{p} / m_{e}\right) \frac{p-2}{p-1}+1, F(x)=x \int_{x}^{\infty} K_{5 / 3}(k) d k$, where $K_{5 / 3}$ is a Bessel function, and $\nu_{c r}^{\prime}=3 \gamma_{e}^{2} e B^{\prime} /\left(4 \pi m_{e} c\right)$. The electron distribution follows a segmented power law, where

$$
\frac{d N_{e}^{\prime}}{d \gamma_{e}} \propto \begin{cases}\gamma_{e}^{-p} & \text { if } \gamma_{e}<\gamma_{c} \\ \gamma_{e}^{-(p+1)} & \text { if } \gamma_{e} \geq \gamma_{c}\end{cases}
$$

Here, $\gamma_{c}=6 \pi m_{e} c /\left(\sigma_{T} \gamma B^{\prime 2} t\right)$ is called the cooling Lorentz factor, meaning that an electron with $\gamma_{e}<\gamma_{c}$ cools slowly and that with $\gamma_{e} \geq \gamma_{c}$ cools rapidly and $\sigma_{T}$ is Thomson's cross section. As usual, we assume that the magnetic energy density is a fraction $\xi_{B}^{2}$ of the energy density, i.e. $B^{\prime 2} / 8 \pi=\xi_{B}^{2} e^{\prime}$, and that the electrons carry a fraction $\xi_{e}$ of the energy. A more detailed description of the electron distribution can be found in Dai et al. (1999) and Huang \& Cheng (2003). 


\section{Numerical Results}

In this section, we calculate the evolution of GRB jets to understand the effects of a density rising region on the observed lightcurves, especially how the parameters of this density rising region (such as $n_{2}, R_{\text {rise }}$, and $d$ ) affect the optical afterglows. Progenitor models predict an ejected mass $M_{\mathrm{ej}} \sim 10^{-7}-10^{-9} M_{\odot}$, here we take $M_{\mathrm{ej}}=10^{-8} M_{\odot}$. For the initial Lorentz factor, the electron power-law index and the equipartition factors, we assume conventional values: $\gamma_{0}=300, p=2.2, \xi_{e}=0.1$, and $\xi_{B}^{2}=10^{-4}$. The crosssectional radius is taken as $a_{0}=10^{14} \mathrm{~cm}$ as a reference value, as Cheng et al. (2001) do. Unless otherwise specified, the parameters illustrated in Figure 1 are set as $n_{1}=10 \mathrm{~cm}^{-3}$, $n_{2}=200 \mathrm{~cm}^{-3}, R_{\text {rise }}=3 \mathrm{pc}$, and $d=1 \mathrm{pc}$. We place ourselves at $z=1$ from the source and right on the jet axis of the cylindrical jet (an on-axis observer with $\Theta=0$ ). In this paper we assume a flat universe with $\Omega_{m}=0.27, \Omega_{\mathrm{vac}}=0.73$ and $H_{0}=71 \mathrm{~km} \mathrm{~s}^{-1} \mathrm{Mpc}^{-1}$, in which case $z=1$ corresponds to a luminosity distance $D_{L} \approx 6.6 \mathrm{Gpc}$. We start our calculations when afterglow phase starts, i.e. $m_{0}=M_{\mathrm{ej}} / \gamma_{0}$. This happens at the deceleration radius $R_{\mathrm{dec}}=m_{0} /\left(\pi a_{0}^{2} n m_{\mathrm{p}}\right)$, about $t_{0}=R_{\mathrm{dec}} /\left(2 c \gamma_{0}^{2}\right)$ after the main burst.

Figure 2 shows the effect of varying $R_{\text {rise }}$ on the lightcurves. A bump in the lightcurve appears when the jetted GRB ejecta meets the boundary of stellar wind and the molecular cloud (Region 2 in Figure 1). Following the bump is a marked decrease of the brightness. The time the bump appears, $t_{\text {rise }}$, is strongly correlated with $R_{\text {rise }}$. Therefore, from the time a bump appeared on an observed GRB lightcurve, we can roughly estimate the distance of the density boundary from the progenitor, provided that other parameters are suitably chosen.

In Figure 3, we show the effect of density $n_{2}$ in Region (3) on the lightcurves. We see that $n_{2}$ affects the shape of the bump in the afterglow lightcurves and the level of the late time flux. The larger $n_{2}$ is, the higher is the bump observed and the shorter is the rising time of the bump.

We vary $d$ in figure 4 to demonstrate the effect of the width of Region (2) on the lightcurves. This time a higher bump and a shorter bump's rising time is caused by a smaller $d$. However, the parameter $d$ cannot affect the level of the late time flux of the 
afterglow.

In Figure 5, the effect of our viewing angle $\Theta$ on the observed afterglow lightcurve is illustrated. As noted by previous authors (e.g. Cheng et al., 2001), a viewing angle larger than $1 / \gamma$ will drastically suppress the early observed flux.

\section{Comparisons of the Model with GRBs 970508, 000301C, and 030226}

\subsection{GRB 970508}

GRB 970508 was detected simultaneously on 1997 May 8 at UT 21:41:50 by the Gammaray Burst Monitor (Costa et al., 1997) and the X-ray Wide Field Camera on BeppoSAX (Jager et al., 1997). Its host galaxy is a starburst galaxy (Sokolov et al., 1999). A high density shell around the GRB progenitor of this burst has been suggested by Piro et al. (1999). They interpreted the dense region to be the ejecta of a preburst supernova. GRB 970508 has a gamma-ray fluence of $3.1 \times 10^{-6} \mathrm{erg} \mathrm{cm}^{-2}$, a redshift $z=0.835$, and a luminosity distance $D_{L}=5.30 \mathrm{Gpc}$, corresponding to an isotropic gamma-ray energy of $E_{\gamma, \text { iso }}=5.68 \times 10^{51} \mathrm{erg}$.

The optical flux remained constant (a plateau) for about 1 day from early observations. Then the $R_{c}$-band flux increases $\sim 1.3 \mathrm{mag}$ in $\approx 1$ day. After that it decayed as a power law in the following tens of days (a possible break may occur around $t=25$ days). This peculiar behavior is puzzling. Panaitescu et al. (1998) and Chang et al. (2002) proposed that delayed energy injection from the central source could account for the bump. Figure 6 shows the observed $R_{c}$-band lightcurve of GRB 970508 and the predictions by the cylindrical jet model using the parameters listed in Table 1. The agreement is very good for over 100 days. Specifically, the early bump at $1-2$ day after the burst can be

satisfactorily reproduced by our model. Thus, the early bump may also be the results of density enhancement in the vicinity of a very massive star in the context of the cylindrical jet model. 


\subsection{GRB $000301 \mathrm{C}$}

GRB 000301C was first detected with both the RXTE All-Sky Monitor and the IPN spacecrafts Ulysses and NEAR on 2000 March 01 at UT 09:51:37 (Smith et al., 2000). The burst has a gamma-ray fluence of $4.1 \times 10^{-6} \mathrm{erg} \mathrm{cm}^{-2}$, a redshift $z=2.033$ and a luminosity distance $D_{L}=16.1 \mathrm{Gpc}$, corresponding to an isotropic gamma-ray energy of $E_{\gamma, \text { iso }}=4.17 \times 10^{52} \mathrm{erg}$. Its infrared/optical afterglow lightcurves show a bump around day 3 and another bump around day 7. The first bump has been interpreted by Garnavich et al. (2000) as due to gravitational microlensing, while other explanations such as density enhancement (Berger et al. 2000) or energy injections have been suggested.

A comparison of the optical data of this burst with our model is shown in Figure 7 using the parameters in Table 1. Our model can reproduce the first bump which appears at around $t \sim 3$ days. This result gives support to a previous study (Berger et al., 2000) that the bump is caused by density enhancement. Here we have only used a simple density profile and we cannot reproduce the two bumps in the lightcurve simultaneously. The two bumps may result from density fluctuations within the high density shell.

\section{$4.3 \quad$ GRB 030226}

The gamma-ray burst GRB 030226 was first detected by the High Energy Transient Explorer 2 satellite on 2003 February 26 at UT 03:46:31.99 (Suzuki et al., 2003). The gamma-ray fluence was $5.7 \times 10^{-6} \mathrm{erg} \mathrm{cm}^{-2}$. The burst has a redshift of $z=1.98$, corresponding to a luminosity distance $D_{L}=15.54 \mathrm{Gpc}$ and an isotropic gamma-ray energy of $E_{\gamma, \text { iso }}=5.53 \times 10^{52} \mathrm{erg}$. Owing to the rapid localization of the burst, Fox et al. (2003) detected an optical counterpart only 0.11 day after the burst. It faded as $t^{-1.2}$ for $\sim 0.2$ day, re-brightened during $0.2-0.5$ day, and finally declined as $t^{-2.0}$, as interpreted by Dai \& Wu (2003). Figure 8 shows the comparison between our model using the numerical values shown in Table 1 and the optical data of this burst.

Although there is no clear bump seen in the lightcurve of this burst, our model really fits very well all data points. We have used the photometric data of Pandey et al. (2004), which do not suffer from possible inhomogeneous photometric calibrations in the GCN 
Circulars.

\subsection{Summary of Comparisons with Models}

From the above fits, we summarize in Table 1 the values of the parameters used in our

calculations together with the deceleration radius $R_{\mathrm{dec}}$. From Table 1 , we see that $R_{\text {rise }}$ ranges from $0.4-22 \mathrm{pc}$. These values are consistent with the radius $(\sim 0.1-10 \mathrm{pc})$ of pressure-driven bubbles created by strong stellar winds of very massive stars at the end of their life (Weaver et al., 1977; Shull, 1980). The values of $n_{1}=1.6-100 \mathrm{~cm}^{-3}$ used in our models are consistent with those calculated by Shull (1980). Note that similar values are inferred by broadband afterglow modeling employing conical jet geometry (Panaitescu \& Kumar, 2001, 2002; Yost et al., 2003). The values we obtained for $n_{2}$, ranging from $n_{2} \sim 10^{2}-10^{3} \mathrm{~cm}^{-3}$, are typical interclump densities in galactic GMCs. The parameter $d$ is the width of the ramping density region from the stellar wind to the molecular cloud. We know little about its "standard" values because this region is highly viable from different works. It is primarily because of different assumptions of the ambient densities of the massive stars and evolutionary trends during the final stages of the massive stars. Specifically, the nice fit of GRB 970508 (and the values of the parameters used in the fit) suggests that the bubble is created by the massive star during its main-sequence stage (see Weaver et al., 1977).

\section{Discussion}

Long-duration GRB progenitors are widely believed to be massive stars because their associations with SNe and star forming regions is clearer than anytime before. Since molecular clouds are the only places where very massive stars are born and die, we believe that GRB progenitors are surrounded by stellar wind and overdense regions resulting from wind interactions with dense molecular clouds. Ramirez-Ruiz et al. (2001) also considered the afterglows of GRBs resulting from such environments. They assumed that the jet of GRB has a conical geometry and they were able to explain the late-time re-brightenings in 
some GRB afterglow lightcurves, such as the bumps observed in afterglows of GRB 970228, GRB 980326, and GRB 000911. However, their model cannot explain the re-brightenings occurred at $t \lesssim 10$ days after the bursts, as seen in the optical lightcurves of GRB 970508 and GRB 000301C.

We will first discuss the homogeneity of Region (1). It is well known that stellar winds blow throughout the entire life of very massive stars. This wind will dramatically affect the GRB environment. Many authors (e.g. Chevalier \& Li, 1999, 2000; Wu et al., 2003) have discussed how a free wind environment (i.e. $n \propto r^{-2}$ ) affects the lightcurves of GRB afterglows. However, the free wind region terminates at a radius much smaller than $10^{16}$ $\mathrm{cm}$ if the star was born in a cloud of $n_{\mathrm{H}} \sim 10^{5} \mathrm{~cm}^{-3}$ (Weaver et al., 1977; Shull, 1980). The simulations by Ramirez-Ruiz et al. (2001) further demonstrated that a region with nearly constant density (or quasi-uniform density) indeed exists between the free wind region and the high density region. Recently, this idea has also been suggested by several other authors (e.g. Chevalier et al., 2004). Berger et al. (2003b) shows that a radio flare from GRB 020405 gives support to the presence of a uniform density medium in the range $R \sim 10^{15}-10^{17} \mathrm{~cm}$.

In this paper, we show the optical afterglow lightcurve signatures of a GRB ejecta meeting a low-to-high density boundary. Our modeled lightcurves are consistent with the afterglows of GRB 970508, GRB 000301C, and GRB 030226. We employ the idea that the boundary is formed by interactions between the preburst strong wind with a dense cloud (Ramirez-Ruiz et al., 2001). It should be noted that other possibilities may give rise to such a boundary as well. For example, interactions between fast and slow winds (Luo \& McCray, 1991; Vikram \& Balick, 1998) or preburst supernova ejecta (Vietri \& Stella, 1998) may also trigger overdense regions (or shells) in the surroundings of GRB progenitors. Thus, we consider such density boundaries very likely to exist along the path of GRB remnants (see e.g. Dai \& Lu, 2002) and our results can also apply to these environments which involve such kind of density boundaries.

Reverse shock may be an important factor in cases when density jump exists. However, in our current study, the reverse shock will not form and can be essentially omitted. The reason is as follows. It is believed that the condition to form a relativistic shock is that 
the density increases abruptly, and that at the same time the density contrast $\left(n_{2} / n_{1}\right)$ must be much larger than 21 (Dai \& Lu, 2002; Dai \& Wu, 2003). If this condition is not satisfied, then the reverse shock is at most Newtonian (In this case, the emission from the Newtonian reverse shock is very week as compared with that from the forward shock, and can essentially be omitted. The dynamics of the flow will not be affected markedly also). In our calculations, the overall density contrast $\left(n_{2} / n_{1}\right)$ is generally less than 21 (see our fits of GRB 000301C and GRB 030226). Additionally, in our model, the density does not increase abruptly, but slowly. That is, the density rises from $n_{1}$ to $n_{2}$ in a distance $d$ which is between $0.5 \mathrm{pc}$ and $5 \mathrm{pc}$. This is a relatively large distance. Thus, the condition for relativistic reverse shock to take effects will not be satisfied in the situations we considered here.

We notice that the afterglow of GRB 030329 also shows an early bump at $1-2$ days. This bump can possibly be interpreted in our framework, although an interpretation using a two-component jet model also seems satisfactory (Berger et al., 2003a).

As a final note, the movement of a massive star during its life may complicate the density distribution arising from the stellar wind and its interaction with the surroundings. A better understanding of the wind environments around very massive stars during the end of their lives is important for a complete description of GRBs and the afterglows.

We would like to thank the anonymous referee for useful comments and suggestions, and $\mathrm{X}$. Y. Wang for valuable discussions and comments. This work is supported by a RGC grant of Hong Kong SAR Government of China under HKU7014/04P.

\section{References}

Ando, M. et al., 2003a. GCN Circ. 1882.

Ando, M. et al., 2003b. GCN Circ. 1884.

Berger, E. et al., 2000. ApJ 545, 56.

Berger, E. et al., 2003a. Nature 426, 154.

Berger, E., Soderberg, A.M., Frail, D.A., \& Kulkarni, S.R., 2003b. ApJ 587, L5.

Biretta, J.A., Sparks, W.B., \& Macchetto, F., 1999. ApJ 520, 621. 
Bloom, J.S., 2003. In: Gamma-Ray Bursts in the Afterglow Era - Third Workshop, ASP

Conf. Series, Vol. 2003, Feroci, M. et al., astro-ph/0303478.

Bloom, J.S. et al., 1999. Nature 401, 453.

Bloom, J.S. et al., 2002. ApJ 572, L45.

Castro-Tirado, A.J. et al., 1998. Science 279, 1011.

Castor, J., McCray, R., \& Weaver, R., 1975. ApJ 200, L107.

Chang, H.Y., Lee, C.H., \& Yi, I., 2002. A\&A 381, L5.

Cheng, K.S., Huang, Y.F., \& Lu, T., 2001. MNRAS 325, 599.

Cheng, K.S., \& Lu, T., 2001. ChJAA 1, 1.

Chevalier, R.A., \& Li, Z.Y., 1999. ApJ 520, L29.

Chevalier, R.A., \& Li, Z.Y., 2000. ApJ 536, 195.

Chevalier, R.A., Li, Z.Y., \& Fransson, C., 2004. ApJ 606, 369.

Costa, E. et al., 1997. IAUC 6649.

Covino, S. et al., 2003. GCN Circ. 1909.

Dai, Z.G., Huang, Y.F., \& Lu, T., 1999. ApJ 520, 634.

Dai, Z.G., \& Lu, T., 2000. ApJ 537, 803.

Dai, Z.G., \& Lu, T., 2002. ApJ 565, L87.

Dai, Z.G., \& Wu, X.F., 2003. ApJ 591, L21.

Esin, A.A., \& Blandford, R., 2000. ApJ 534, L151.

Fatkhullin, T., Komarova, V., Sokolov, V., Cherepashchuk, A., \& Postnov, K., 2003.

GCN Circ. 1925.

Fendt, C., \& Ouyed, R., 2004. ApJ 608, 378.

Frail, D.A. et al., 2001. ApJ 562, L55.

Fox, D.W., Chen, H.W., \& Price, P.A., 2003. GCN Circ. 1879.

Galama, T.J. et al., 1998. ApJ 497, L13.

Galama, T.J. et al., 2000. ApJ 536, 185.

Galama, T.J., \& Wijers, R.A.M.J., 2001. ApJ 549, L209.

Garnavich, P.M., Loeb, A., \& Stanek, K.Z., 2000. ApJ 544, L11.

Garnavich, P.M., von Braun, K., \& Stanek, K.Z., 2003a. GCN Circ. 1885.

Garnavich, P.M. et al., 2003b. ApJ 582, 924.

Greiner, J., Ries, C., Barwig, H., Fynbo, J., \& Klose, S., 2003. GCN Circ. 1894. 
Guarnieri A. et al., 2003. GCN Circ. 1892.

Hjorth, J. et al., 2003. Nature 423, 847.

Holland, S., \& Hjorth, J., 1999. A\&A 344, L67.

Holland, S. et al., 2001. A\&A 371, 52.

Huang, Y.F., \& Cheng, K.S., 2003. MNRAS 341, 263.

Huang, Y.F., Dai, Z.G., \& Lu, T., 1999a. Chin. Phy. Lett. 16, 775.

Huang, Y.F., Dai, Z.G., \& Lu, T., 1999b. MNRAS, 309, 513.

Huang, Y.F., Dai, Z.G., \& Lu, T., 2000a. MNRAS, 316, 943.

Huang, Y.F., Dai, Z.G., \& Lu, T., 2000b. A\&A 355, L43.

Huang, Y.F., Gou, L.J., Dai, Z.G., \& Lu, T., 2000c. ApJ 543, 90.

Huang, Y.F., Tan, C.Y., Dai, Z.G., \& Lu, T., 2002. ChA\&A 26, 414.

Jager, R. et al., 1997. A\&AS 125, 557.

Krasnopolsky, R., Li, Z.Y., \& Blandford, R.D., 2003. ApJ 595, 631.

Lamb, D.Q., \& Reichart, D.E., 2000. ApJ 536, 1.

Luo, D., \& McCray, R., 1991. ApJ 379, 659.

Ma, H.T., Huang, Y.F., Dai, Z.G., \& Lu, T., 2003. ChJAA 3, 225.

Maiorano, E. et al., 2003. GCN Circ. 1933.

Masetti, N. et al., 2000. A\&A 359, L23.

Nysewander, M. C., Moran, J., Reichart, D., Henden, A., \& Schwartz, M., 2003. GCN

Circ. 1921.

Paczyński, B., 1998. ApJ 494, L45.

Panaitescu, A., \& Kumar, P., 2001. ApJ 560, L49.

Panaitescu, A., \& Kumar, P., 2002. ApJ 571, 779.

Panaitescu, A., Mészáros, P., \& Rees, M.J., 1998. ApJ 503, 314.

Pandey, S.B. et al., 2004. A\&A 417, 919.

Perdersen, H. et al., 1998. ApJ 496, 311.

Perley, R.A., Bridle, A.H., \& Willis, A.G., 1984. ApJS 54, 291.

Piran, T., 1999. Phys Rep. 314, 575.

Piro, L. et al., 1999. ApJ 514, L73.

Price, P.A., \& Warren, B.E., 2003. GCN Circ. 1890.

Ramirez-Ruiz, E., Dray, L.M., Madau, P., \& Tout, C.A., 2001. MNRAS 327, 829. 
Ray, T.P. et al., 1996. ApJ 468, L103.

Reichart, D.E., \& Price, P.A., 2002. ApJ 565, 174.

Reichart, D.E., \& Yost, S.A., 2001. ApJ submitted, astro-ph/0107545.

Rhoads, J.E., 1997. ApJ 487, L1.

Rhoads, J.E., 1999. ApJ 525, 737.

Rhoads, J.E., \& Frutchter, A.S., 2001. ApJ 546, 117.

Rumyantsev, V., Biryukov, V., \& Pozanenko, A., 2003a. GCN Circ. 1908.

Rumyantsev, V., Biryukov, V., \& Pozanenko, A., 2003b. GCN Circ. 1929.

Rybicki, G.B., \& Lightman, A.P., 1979. Radiative Processes in Astrophysics, New York:

Willey.

Sagar, R., Mohan, V., Pandey, S.B., Pandey, A.K., Stalin, C.S., \& Castro-Tirado, A.J., 2000. BASI 28, 499, astro-ph/0004223

Sari, R., Piran, T., \& Halpern, J.P., 1999. ApJ 519, L17.

Schlegel, D.J., Finkbeiner, D.P., \& Davis, M., 1998. ApJ 500, 525.

Semkov, E.H., 2003. GCN Circ. 1935.

Shu, F.H. et al., 1995. ApJ 455, L155.

Shull, J.M., 1980. ApJ 238, 860.

Sokolov, V.V., 1999. A\&A 344, 43.

Solomon, P.M., Rivolo, A.R., Barrett, J., \& Yahil, A., 1987. ApJ 319, 730.

Suzuki, M. et al., 2003. GCN Circ. 1888.

Vietri, M., \& Stella, L., 1998. ApJ 507, L45.

Vikram, D., \& Balick, B., 1998. ApJ 497, 267.

Vlahakis, N., \& Königl, A., 2003a. ApJ 596, 1080.

Vlahakis, N., \& Königl, A., 2003b. ApJ 596, 1104.

von Braun, K., Garnavich, P., \& Stanek, K., 2003. GCN Circ. 1881.

Wang, X.Y., Dai, Z.G., \& Lu, T., 2000. MNRAS 317, 170.

Weaver, R., McCray, R., Castor, J., Shapiro, P., \& Moore, R., 1977. ApJ 218, 377.

Woosley, S.E., 1993. ApJ 405, 273.

Wu, X.F., Dai, Z.G., Huang, Y.F., \& Lu, T., 2003. MNRAS 342, 1131.

Yost, S.A., Harrison, F.A., Sari, R., \& Frail, D.A., 2003. ApJ 597, 459. 


\begin{tabular}{|lccccccccccc|}
\hline $\begin{array}{c}R_{\text {rise }} \\
\text { GRB }\end{array}$ & $\begin{array}{c}n_{1} \\
(\mathrm{pc})\end{array}$ & $\begin{array}{c}\mathrm{cm}_{2} \\
\left(\mathrm{~cm}^{-3}\right)\end{array}$ & $\left(\mathrm{cm}^{-3}\right)$ & $(\mathrm{pc})$ & $\left(10^{-8} M_{\odot}\right)$ & $p$ & $\xi_{B}^{2}$ & $\xi_{e}$ & $(\mathrm{rad})$ & $\left(10^{17} \mathrm{~cm}\right)$ \\
\hline \hline 970508 & 22 & 1.6 & 100 & 5 & 26 & 2.0 & $10^{-4}$ & 0.1 & 0.006 & $2.1 \times 10^{2}$ \\
\hline $000301 \mathrm{C}$ & 2.95 & 10 & 60 & 1.5 & 0.19 & 2.5 & $10^{-3}$ & 0.01 & 0.01 & 0.23 \\
\hline 030226 & 0.4 & 100 & 1200 & 0.5 & 0.80 & 2.1 & $1.5 \times 10^{-5}$ & 0.03 & 0 & 0.10 \\
\hline
\end{tabular}

Table 1: The parameters used in our model fits to optical afterglow lightcurves of GRBs $970508,000301 \mathrm{C}$, and 030226

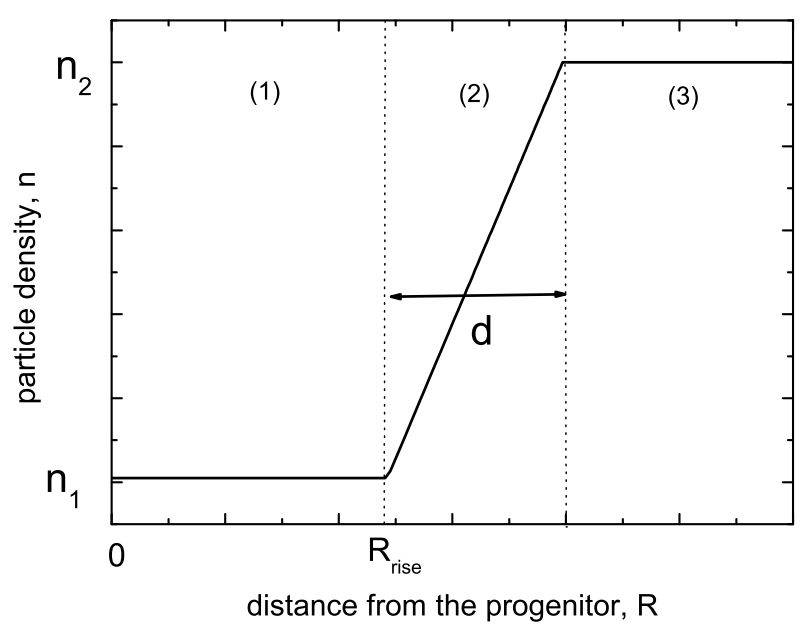

Figure 1: The assumed density profile of the GRB system inside a molecular cloud. The density in Region (1) is a constant $n_{1}$ while the density in Region (3) is $n_{2}$. Region (2) is an intervening region at a distance $R_{\text {rise }}$ from the progenitor of width $d$. 


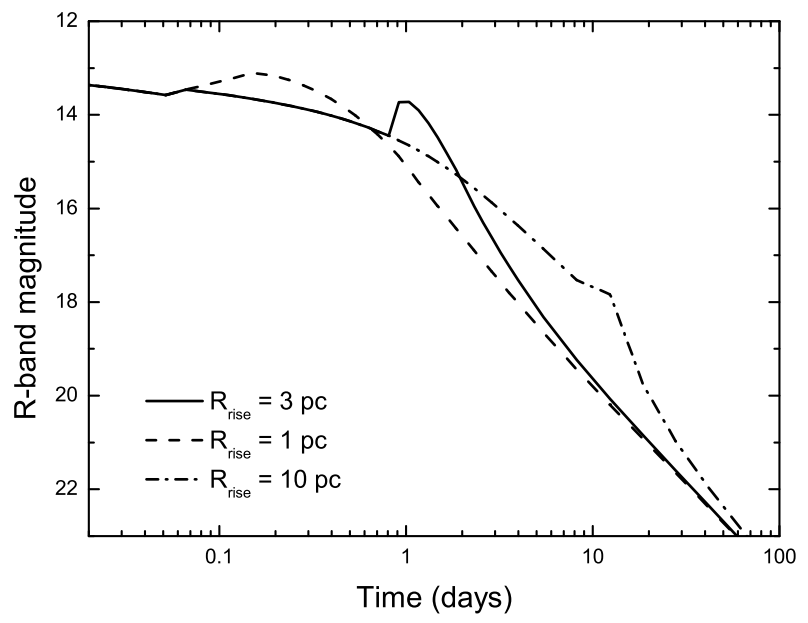

Figure 2: Simulated optical lightcurves from our model. Except for $R_{\text {rise }}$, other parameters are kept constant (see text). 


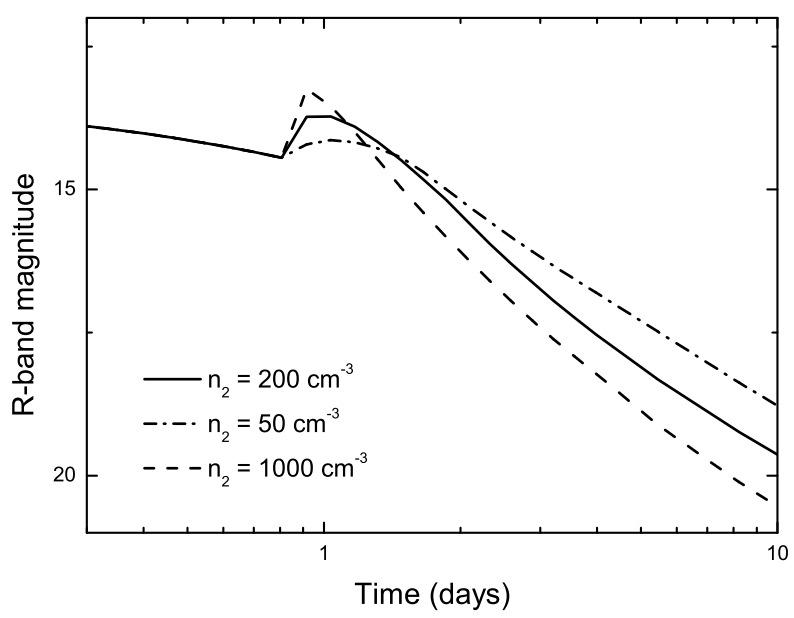

Figure 3: Simulated optical lightcurves from our model. Except for $n_{2}$, other parameters are kept constant (see text).

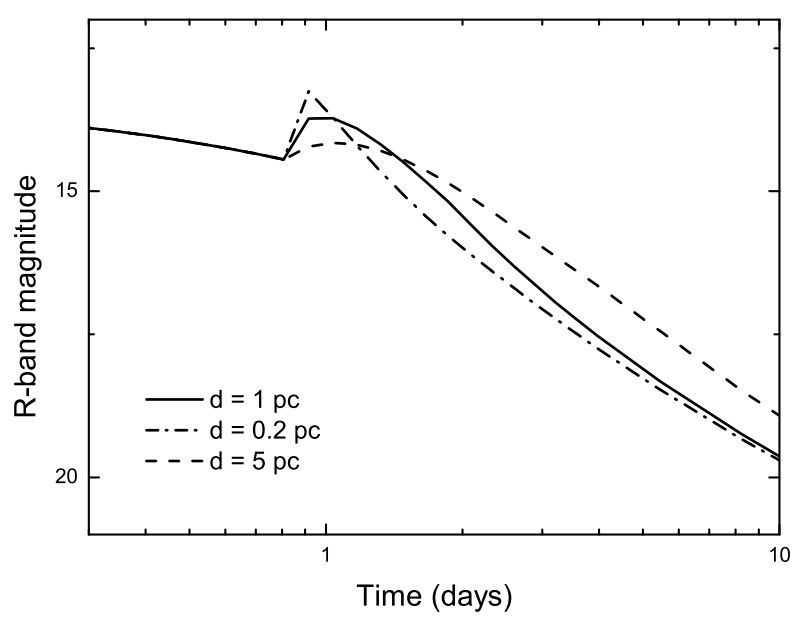

Figure 4: Simulated optical lightcurves from our model. Except for $d$, other parameters are kept constant (see text). 


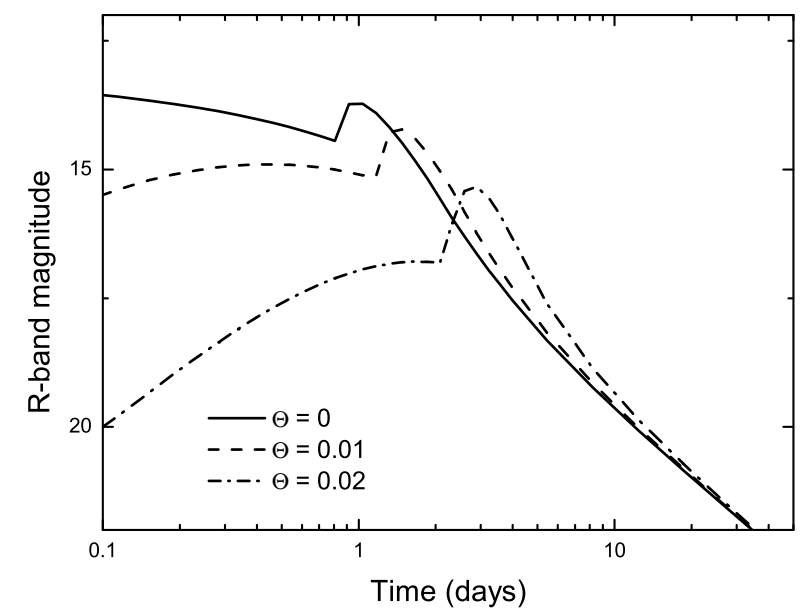

Figure 5: Simulated optical lightcurves from our model. Except for $\Theta$, other parameters are kept constant (see text).

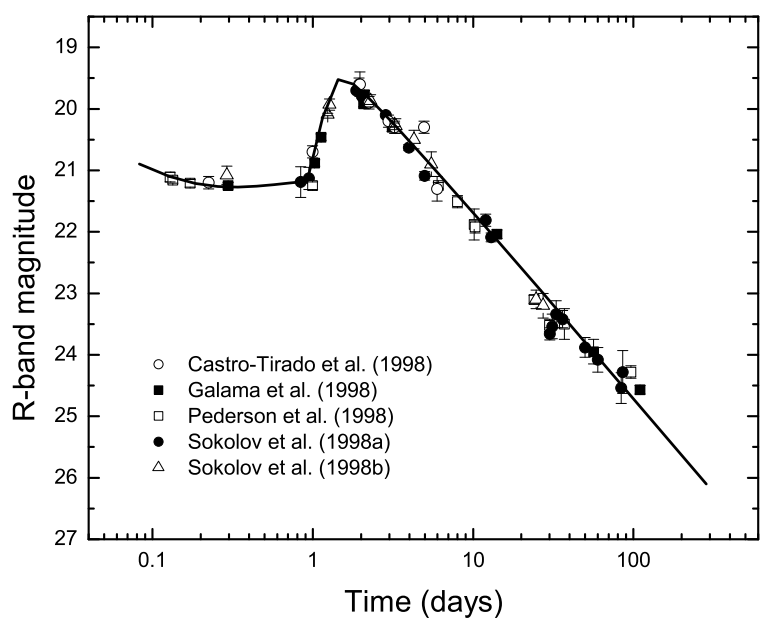

Figure 6: Comparison of the model with GRB $970508 R_{c}$-band afterglow lightcurve. Data points are from Castro-Tirado et al. (1998, empty circles), Galama et al. (1998, filled squares), Perdersen et al. (1998, empty squares) and Sokolov et al. (1998a, filled circles; 1998b, empty triangles). The error bar of the latest data point is too small to be shown in the figure. 


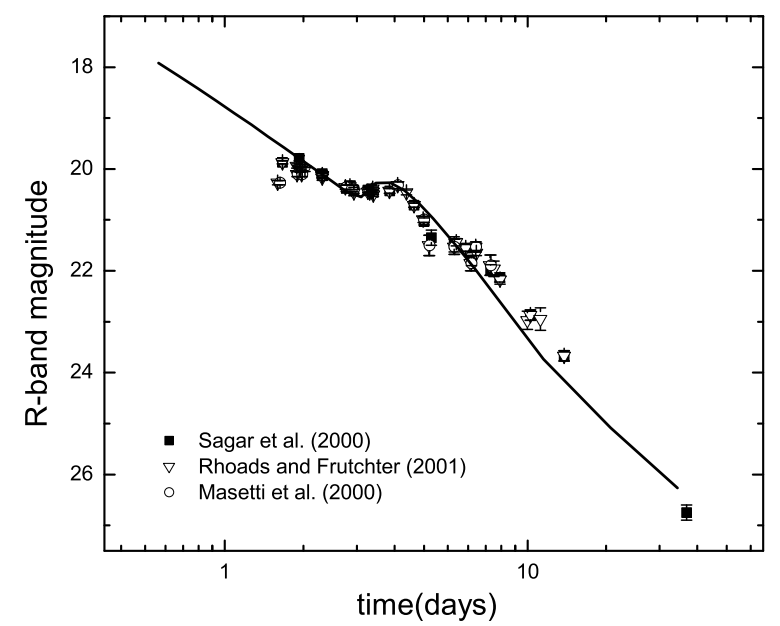

Figure 7: Comparison of the model with GRB 000301C $R_{c}$-band afterglow lightcurve. Data points are from Masetti et al. (2000, empty circles), Rhoads \& Frutchter (2001, empty upper triangles) and Sagar et al. (2000, filled squares), corrected for Galactic foreground extinction $(R+15 \%$; Schlegel et al., 1998). 


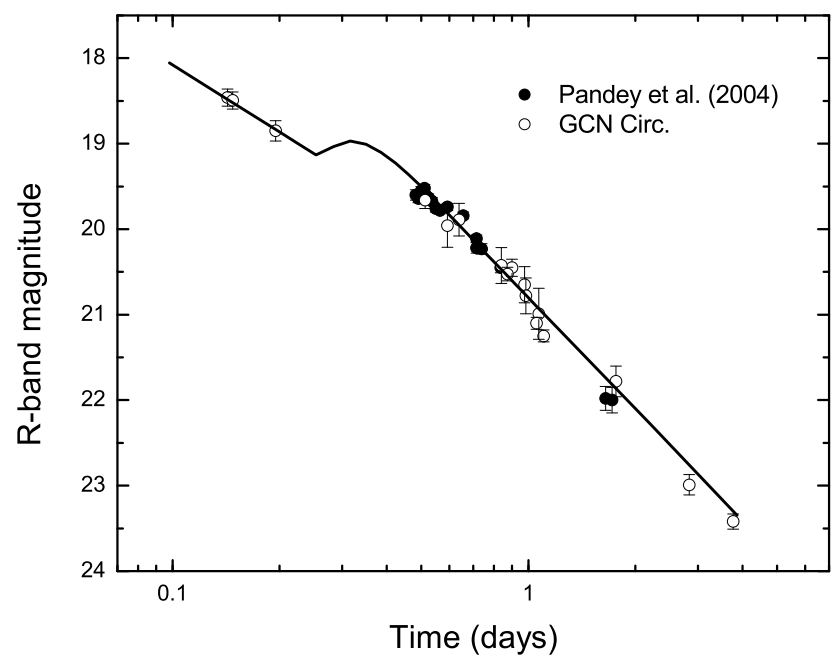

Figure 8: Comparison of the model with GRB $030226 R_{c}$-band afterglow lightcurve. Data points are from Pandey et al. (2004; filled circles) and the following GCN Circ. (empty circles): Ando et al. (2003a, 2003b), Covino et al. (2003), Fatkhullin et al. (2003), Garnavich et al. (2003a), Greiner et al. (2003), Guarnieri et al. (2003), Maiorano et al. (2003), Nysewander et al. (2003), Price \& Warren (2003), Rumyantsev et al. (2003a, 2003b), Semkov (2003) and von Braun et al. (2003). 